\title{
Envelhecimento e capacidade para o trabalho dos trabalhadores de higiene e limpeza hospitalar*
}

\author{
AGING AND WORK ABILITY OF WORKERS OF A UNIVERSITY HOSPITAL'S CLEANING \\ AND HYGIENE SERVICE
}

\section{ENVEJECIMIENTO Y CAPACIDAD PARA EL TRABAJO DE LOS EMPLEADOS DE HIGIENE Y LIMPIEZA HOSPITALAR}

\section{Cristiane Batista Andrade', Maria Inês Monteiro²}

\section{RESUMO}

As mudanças demográficas ocorridas no Brasil mostram um aumento da população que está envelhecendo e em idade produtiva. Estudo com trabalhadores do Serviço de Higiene e Limpeza de um hospital universitário, não terceirizado, teve por objetivo avaliar a capacidade para o trabalho. Foi utilizado o Índice de Capacidade para o Trabalho, instrumento autoaplicável, desenvolvido por pesquisadores finlandeses. Foram entrevistados 69 trabalhadores: 21,7\% tinham ótima capacidade para o trabalho; $31,9 \%$ boa; $31,9 \%$ moderada e $14,5 \%$, baixa. As doenças com diagnóstico médico mais freqüientes foram as lesões por acidentes, músculo-esqueléticas e cardiovasculares. O grupo etário de 50 a 60 anos obteve menor Índice de Capacidade para o Trabalho e maior número de doenças, afetando, portanto, a capacidade para o trabalho. Sendo assim, há necessidade do desenvolvimento de programas de promoção à saúde, tendo em vista a recuperação e manutenção da capacidade para o trabalho.

\section{DESCRITORES}

Envelhecimento.

Trabalho.

Avaliação da capacidade

de trabalho.

Serviço hospitalar de limpeza.

\section{ABSTRACT}

This study was conducted with the cleaning personnel of a university hospital in the city of Campinas, State of São Paulo. These are public employees whose work is mainly physical: cleaning is a job that requires heavy physical work; in addition, workers are also exposed to chemical products. The objective of this survey was to evaluate the work ability of cleaners from different age groups, for which was used the Work Ability Index, an instrument developed by Finnish researchers. The sample was comprised of 69 workers who answered the questionnaire: $21.7 \%$ have excellent work ability; $31.9 \%$ good; $31.9 \%$ moderate; and $14.5 \%$ poor. The most frequently reported diseases with medical diagnosis were: lesion due to accidents, musculoskeletal disorders, and cardiovascular diseases. The members of the older group ranked lower in the Work Ability Index and reported a larger number of diseases, which therefore affected their work ability.

\section{RESUMEN}

El estudio ha sido hecho con trabajadores del Servicio de Higiene y Limpieza de un hospital universitario, ya que son empleados públicos y no son de empresas de terceros. Este estudio tuvo como objetivo evaluar la capacidad para el trabajo de estos funcionarios con distintas edades. Se ha utilizado el ICT (Índice de Capacidad para el Trabajo), instrumento autoaplicable, desarrollado por investigadores finlandeses. La población ha sido compuesta por 69 trabajadores, siendo que el $21,7 \%$ tienen óptima capacidad para el trabajo; el 31,9\% buena; el $31,9 \%$ moderada y el $14,5 \%$, baja. Las enfermedades con diagnostico médico más frecuente han sido las lesiones por accidentes, las músculoesqueléticas y las cardiovasculares. El grupo de más edad, de 50 a 60 anos, tuvo menor índice de capacidad para el trabajo y mayor número de enfermedades.

\section{KEY WORDS}

Ageing.

Work.

Work capacity evaluation.

Housekeeping, hospital.

\author{
DESCRIPTORES \\ Envejecimiento. \\ Trabajo. \\ Evaluación de capacidad \\ de trabajo. \\ Servicio de limpieza en hospital.
}

\footnotetext{
* Extraído da dissertação "Envelhecimento e capacidade para o trabalho dos trabalhadores do serviço de higiene e limpeza de um hospital universitário". Faculdade de Ciências Médicas da (FCMUNICAMP), 2002. 1 Mestre em

Enfermagem. Doutoranda em Educação. Professora de Qualidade de Vida no Trabalho - curso de Administração da Faculdade de Jaguariúna-SP e Docência em Fundamentos Sociológicos na Educação do curso de Pedagogia (PROESF) da Unicamp. criks@yahoo.com

2 Professora

Associada do Departamento de Enfermagem da FCM Universidade Estadual de Campinas. Orientadora da Dissertação. Departamento de Enfermagem da FCM - UNICAMP. inesmon@ fcm.unicamp.br
} 


\section{INTRODUÇÃO}

O interesse em estudar o envelhecimento e a capacidade para o trabalho dos trabalhadores dos Serviços Gerais de um hospital universitário surgiu da necessidade de aprofundar o tema, visto que, em estudo anterior, verificamos que os mesmos têm baixa escolaridade, baixos rendimentos, possuem problemas de saúde relacionados ao trabalho (doenças osteomusculares relacionadas ao trabalho, dorsocervicolombalgias, estresse e outras), estão expostos a riscos químicos, desenvolvem atividades que requerem esforço físico, além de terem uma inserção precoce no mercado de trabalho ${ }^{(1)}$.

É importante considerar, que paralelamente a essas particularidades de trabalho dessa categoria estudada, o Brasil vivencia o processo de transição demográfica, caracterizado pelo aumento do número de pessoas que estão envelhecendo, ocasionando, portanto, o envelhecimento da população em idade produtiva e também da força de trabalho.

Esse processo que os países estão enfrentando é consequiência da diminuição nas taxas de natalidade, mortalidade infantil e das doenças infecciosas, levando a um aumento da esperança de vida, do número de pessoas idosas e da população em idade produtiva ${ }^{(2)}$.

Além desse processo de envelhecimento populacional, há na realidade brasileira, trabalhadores com inserção precoce no mercado de trabalho, além das condições de vida, saúde e trabalho não serem adequadas para um grande contingente da população.

Portanto, é relevante a realização de estudos da relação trabalho-saúde para que sejam desenvolvidas medidas de promoção à saúde, à população em idade produtiva que está envelhecendo, tendo em vista a preservação da capacidade para o trabalho e a manutenção da qualidade de vida dentro e fora do trabalho.

A capacidade para o trabalho está diretamente relacionada com a possibilidade de realizar atividades que envolvam as aptidões físicas, mentais, funcionais e sociais ${ }^{(3)}$.

A maior parte dos estudos realizados sobre capacidade para o trabalho tem sido desenvolvida na Finlândia, e os resultados apontam que esse processo sofre influências das condições de trabalho e de saúde, do estilo de vida e do envelhecimento biológico, pois, de acordo com esse processo, há diminuição dos movimentos das articulações, perda da força, resistência muscular e elasticidade dos tecidos, aumento das dorsocervicolombalgias e diminuição das tomadas de decisões ${ }^{(4-5)}$.
Sendo assim, a manutenção da capacidade para o trabalho envolve condições de saúde e de trabalho adequadas, quer sejam as de relações interpessoais ou ambientais. É importante ressaltar que dessa forma, isso se traduzirá em uma melhor qualidade de vida dentro e fora do trabalho, numa maior produtividade e num período de aposentadoria mais proveitoso. Além de diminuir custos e gastos com o setor público de saúde e de previdência social ${ }^{(5)}$.

Diante disso, é relevante atentar para os trabalhos que têm como característica a exigência de esforço muscular, como no caso de trabalhadores de higiene e limpeza, pois realizam levantamento e transporte de peso, esforços repetitivos e repentinos, têm posturas de trabalho inadequadas, inclinação simultânea, sobrecarga postural e do sistema músculo-esquelético, além de riscos de acidente de trabalho e exposição a produtos químicos, e necessitam de medidas de promoção à saúde, visto a possibilidade de perda da capacidade para o trabalho dos trabalhadores inseridos nessas atividades profissionais ${ }^{(3)}$.

Alguns estudos realizados com trabalhadores de higiene e limpeza mostram que há consideráveis problemas de saúde relacionados ao trabalho como: dermatológicos, respiratórios, reprodutivos, câncer, contaminação biológica (hepatite A, B e C, tuberculose), doenças músculoesqueléticas e na capacidade física ${ }^{(6)}$.

Sem dúvida, problemas de saúde relacionados ao trabalho são comuns entre esses trabalhadores, e a capacidade para o trabalho precisa ser preservada. Para que isso ocorra, o compromisso com a promoção à saúde deve acontecer conjuntamente com outros setores, além da saúde, como as organizações não governamentais e voluntárias, setores social e governamental e sindicatos.

A Fundação Européia para o Melhoramento das Condições de Vida e de Trabalho, de Dublin, realizou estudos sobre o envelhecimento e a capacidade para o trabalho, visando a implementação de programas relativos a reorganização do trabalho (adequação do ambiente, equipamentos e métodos de trabalho); redução e flexibilidade dos horários de trabalho; implementação de programas de atividades físicas e de preparação para a aposentadoria; atividades de incentivo à criatividade e ao crescimento pessoal, dentre outros, tendo em vista a necessidade de preservação da capacidade para o trabalho ${ }^{(5)}$.

Diante do exposto, o objetivo geral desse estudo foi avaliar a capacidade para o trabalho dos trabalhadores do Serviço de Higiene e Limpeza de um hospital universitário, tendo como objetivos específicos avaliar a capacidade para o trabalho em diferentes faixas etárias utilizando o Índice de Capacidade para o Trabalho (ICT), e descrever as doenças auto-referidas e as com diagnóstico médico. 


\section{MÉTODO}

\section{Sujeitos}

Esse estudo foi desenvolvido junto aos trabalhadores permanentes, lotados no Setor de Serviços Gerais especificamente os que atuam no setor de limpeza hospitalar - de um hospital universitário, na cidade de Campinas, Estado de São Paulo.

A opção por desenvolver o estudo com estes trabalhadores ocorreu pelo fato de que no Brasil, as condições de saúde e de trabalho são, em alguns setores, precárias. Além do fato que os trabalhadores da limpeza hospitalar do estudo em questão, têm uma inserção precoce no mercado de trabalho, por isso, o estudo da capacidade para o trabalho e a promoção à saúde desses trabalhadores ainda jovens é importante, para que seja realizada a avaliação da capacidade para o trabalho, tendo em vista a sua manutenção e/ou restauração.

\section{Instrumento para a coleta de dados}

Para avaliar a capacidade para o trabalho e descrever as doenças referidas na própria opinião e as com diagnóstico médico foi utilizado o Índice de Capacidade para o Trabalho (ICT), instrumento auto-aplicável que foi desenvolvido na Finlândia, traduzido para a versão em português, no Brasil.

A validação desse instrumento foi realizada por autores finlandeses entre 1981 e $1982^{(7)}$.

O instrumento permite avaliar a capacidade para o trabalho atual comparada com o melhor de toda a vida, a capacidade para o trabalho em relação às exigências físicas e mentais, número atual de doenças auto-referidas e as com diagnóstico médico, impedimento para o trabalho devido às doenças, faltas no trabalho por doenças nos últimos doze meses, prognóstico próprio sobre a capacidade para o trabalho daqui a dois anos, e capacidade de apreciar as atividades diárias, sentir-se ativo e alerta, e com esperança para o futuro ${ }^{(7)}$.

\section{Coleta de dados}

Foi solicitado ao Setor de Recursos Humanos da instituição, em maio de 2000, uma listagem com o número de funcionários, data de nascimento, local e horário de trabalho e data de ingresso no trabalho. Nesta época, atuavam no referido setor 145 funcionários públicos concursados, sendo que todos tinham mais de 20 anos de idade.

No entanto, no período de coleta de dados, diversos trabalhadores haviam sido remanejados para outros locais de trabalho, em conseqüência do processo de terceirização desse serviço. Portanto, o estudo teve a participação de 72 trabalhadores, sendo que dois questionários estavam incompletos e uma trabalhadora entregou o questionário após o encerramento da coleta de dados. Houve quatro recusas, quatro trabalhadores estavam em férias, sendo que três deles ficaram afastados após o término destas. Deste modo, o número total de sujeitos foi de 69 trabalhadores.

A opção pela não identificação dos sujeitos teve como objetivo assegurar o caráter confidencial dos dados.

O preenchimento do questionário foi realizado durante o horário de trabalho e no próprio local de trabalho. Inicialmente, foi solicitado às supervisoras do Serviço de Higiene e Limpeza, o nome dos trabalhadores presentes no dia da coleta de dados para que pudessem ser localizados.

Para alguns trabalhadores foi necessário assistência no preenchimento do questionário, haja vista que não possuíam facilidade na leitura e na compreensão do mesmo, no entanto, a grande maioria conseguiu preenchêlo. No período de preenchimento do questionário a pesquisadora permaneceu junto aos trabalhadores para o esclarecimento de qualquer dúvida.

\section{Aspectos éticos}

Em relação aos aspectos éticos, o projeto de pesquisa foi encaminhado ao Comitê de Ética em Pesquisa (processo 256/200), tendo sido aprovado. Para a realização do termo de consentimento foi utilizado os Requisitos mínimos de protocolo de pesquisa, fundamentado na Resolução 196/96 do Conselho Nacional de Saúde.

\section{APRESENTAÇÃO DOS RESULTADOS}

\section{Tratamento estatístico}

Esse estudo é de caráter epidemiológico transversal ou de prevalência ${ }^{(8)}$. Os dados foram analisados estatisticamente, sendo utilizado o programa SAS System for Windows (Statistical Analysis System) versão 8.1.

Foi realizada a análise descritiva dos dados de variáveis categóricas e das medidas de posição e dispersão das variáveis contínuas. Para verificar associação ou comparar proporções foi utilizado o teste qui-quadrado, quando necessário. Para comparar variáveis contínuas ou ordenáveis entre dois grupos foi aplicado o teste de Mann-Whitney. Para três ou mais grupos, foi utilizado o teste de Kruskal-Wallis. Para verificar a associação linear entre duas variáveis contínuas foi utilizado o coeficiente de correlação de Spearman. O nível de significância adotado foi de $5 \%$. 
O Serviço de Higiene e Limpeza Hospitalar possuía trabalhadores que eram supervisores, portanto, no cálculo dos pontos do ICT , a demanda foi considerada como mental. O cálculo ICT dos auxiliares de limpeza hospitalar foi baseado em demandas predominantemente físicas.

A Tabela 1 a seguir mostra algumas características dos trabalhadores estudados.

Tabela 1 - Freqüência de algumas características dos trabalhadores do Serviço de Higiene e Limpeza de um Hospital Universitário - Campinas - 2001

\begin{tabular}{|c|c|c|c|}
\hline Variáveis & Categtoria & $\mathbf{N}$ & $\%$ \\
\hline \multirow[t]{2}{*}{ Sexo } & Feminino & 62 & 89,9 \\
\hline & Masculino & 07 & 10,1 \\
\hline \multirow{3}{*}{ Faixa etária (anos) } & $30 \nmid 40$ & 05 & 07,2 \\
\hline & $40 \nmid 50$ & 31 & 44,9 \\
\hline & $\geq 50$ & 33 & 47,8 \\
\hline \multirow{4}{*}{ Estado civil } & Casado/vive com companheiro & 40 & 58,8 \\
\hline & Divorciado/separado & 09 & 13,2 \\
\hline & Solteiro & 12 & 17,6 \\
\hline & Viúvo & 07 & 10,3 \\
\hline \multirow{3}{*}{ Categoria Profissional } & Assistente de Serviço & 3 & 04,3 \\
\hline & Auxiliar de Limpeza Hospitalar & 59 & 85,5 \\
\hline & Supervisor & 07 & 10,1 \\
\hline \multirow{5}{*}{ Escolaridade } & Nunca estudou & 02 & 02,9 \\
\hline & Ensino fundamental incompleto ( $1^{\mathrm{a}}$ a $3^{\mathrm{a}}$ série) & 16 & 23,5 \\
\hline & Ensino fundamental incompleto ( $4^{\underline{a}}$ série) & 31 & 45,6 \\
\hline & Ensino fundamental completo & 15 & 22,1 \\
\hline & Ensino médio completo & 04 & 05,9 \\
\hline \multirow{2}{*}{$\begin{array}{l}\text { Realização de tarefas } \\
\text { domésticas }\end{array}$} & Não & 10 & 14,5 \\
\hline & Sim & 59 & 85,5 \\
\hline \multirow{2}{*}{$\begin{array}{l}\text { Outro emprego } \\
\text { remunerado }\end{array}$} & Sim & 66 & 95,7 \\
\hline & Não & 03 & 04,3 \\
\hline \multirow[t]{2}{*}{ Cargo de chefia } & Não & 62 & 89,9 \\
\hline & Sim & 07 & 10,1 \\
\hline
\end{tabular}

Os trabalhadores que possuíam maior nível de escolaridade, geralmente eram supervisores. É importante salientar que todos os supervisores eram do sexo feminino.

Os trabalhadores que atuavam no Serviço de Higiene e Limpeza eram auxiliares de limpeza hospitalar, o que correspondeu a $85,5 \%$ da população, $10,1 \%$, super-visores e apenas 4,3\% eram assistentes de serviço.
A maioria da população estudada, ou seja, $95,7 \%$ não realizava outra atividade remunerada e somente $4,3 \%$ (três trabalhadoras) desenvolviam atividades como diaristas e faxineiras, semelhantes ao tipo de trabalho que exerciam no Serviço de Higiene e Limpeza. Com relação ao desenvolvimento de tarefas domésticas, $85,5 \%$ as realizavam e $14,5 \%$ disseram não executá-las.

A Tabela 2 mostra a distribuição da população segundo o ICT. 
Tabela 2 - Distribuição dos trabalhadores estudados segundo o Índice de Capacidade para o Trabalho - Campinas - 2001

\begin{tabular}{lcc}
\hline Capacidade para o trabalho & $\mathbf{N}$ & $\%$ \\
\hline Baixa (7-27) & 10 & 14,5 \\
Moderada (28-36) & 22 & 31,9 \\
Boa (37-43) & 22 & 31,9 \\
Ótima (44-49) & 15 & 21,7 \\
\hline Total & 69 & 100 \\
\hline
\end{tabular}

Na população estudada, $31,9 \%$ dos trabalhadores tiveram a capacidade para o trabalho pertencente à categoria moderada, $31,9 \%$ à categoria boa, $21,7 \%$ à ótima e $14,5 \%$ na categoria baixa (Tabela 2).

O grupo de doenças com diagnóstico médico predominante foi o das lesões causadas por acidentes, abrangendo $47,8 \%$ da população, seguido pelas cardiovasculares com $40,6 \%$; as músculo-esqueléticas, com $34,8 \%$ e as digestivas, com $21,7 \%$.

Em relação às doenças auto-referidas o grupo predominante foi o das músculo-esqueléticas, com 23,2\% da população; em seguida, as doenças cardiovasculares, respiratórias e as emocionais.

Com relação ao gênero e a capacidade para o trabalho $14,3 \%$ dos homens e $58 \%$ das mulheres estavam nas categorias ótima e boa, e $85,8 \%$ dos homens e $41,9 \%$ das mulheres estavam nas categorias moderada e baixa.

No grupo de 30-50 anos, a média do ICT foi de 38,2 e desvio padrão de 7,5. O grupo com 50-60 anos obteve média do ICT de 34,1 e desvio padrão de 8,4.

Entre os trabalhadores da faixa etária de 30 a 49 anos, $63,9 \%$ estavam na categoria ótima e boa, e 30,7\% nas moderada e baixa. O grupo etário de 50 a 60 anos tinha $33,3 \%$ nas categorias ótima e boa, e $66,7 \%$ nas moderada e baixa.

Com relação à distribuição do ICT e o número de doenças com diagnóstico médico, tem-se que a média de doenças entre os trabalhadores que tiveram ótima capacidade para o trabalho foi de uma doença; boa, duas doenças; moderada, sete doenças e baixa, nove.

\section{DISCUSSÃO DOS RESULTADOS}

Os dados sobre a capacidade para o trabalho entre os trabalhadores estudados mostraram que um número considerável de trabalhadores, ou seja, $46,4 \%$ possuíam a capacidade para o trabalho nas categorias moderada e baixa.
Ao discutir esse dado foi imprescindível, portanto, considerar que a população estudada tinha como característica de trabalho a predominância de demanda física em sua atividade.

Há de salientar também, que os trabalhadores estudados eram funcionários públicos, estando há 14,7 anos, em média, na mesma função, ou seja, no serviço de higiene e limpeza. Em meados de 1986, teve início o funcionamento do hospital universitário estudado, o que permite dizer que muitos trabalhadores entrevistados iniciaram o trabalho nesse período.

Esses achados se assemelham ao de outros autores ${ }^{(9)}$ no qual os trabalhadores estudados, que exerciam demandas físicas de trabalho, tiveram a capacidade para o trabalho diminuída ao longo dos anos, devido a organização e o ambiente de trabalho. Foi constatado que $82 \%$ da população tiveram a capacidade para o trabalho diminuída e somente 13,6\% aumentada, no período de 1982-1991.

Sendo assim, para esses trabalhadores, a recomendação é que a capacidade para o trabalho precisa ser melhorada ou restaurada, a fim de promover a sua saúde. Estudos apontam que a capacidade para o trabalho deverá ser restaurada ou mantida através da atividade física, que proporciona a oxigenação em nível celular, diminui o estresse e melhora a auto-estima. Destacamos, entretanto, que as atividades físicas deverão ser adequadas à idade e, é importante considerar, que esses trabalhadores já realizam atividades predominantemente físicas no trabalho ${ }^{(4)}$.

Uma outra possibilidade em relação à manutenção da capacidade para o trabalho, é através da reorganização do trabalho, remanejando os trabalhadores com capacidade para o trabalho baixa ou moderada, para locais onde possam realizar suas atividades, de acordo com as suas possibilidades físicas e mentais, evitando, pois, o comprometimento da capacidade para o trabalho ${ }^{(4)}$.

Com relação à capacidade para o trabalho - exigências mentais $-92,7 \%$ dos trabalhadores estavam nas categorias muito boa e boa. Esse resultado apontou que, embora a 
população estudada realizasse predominantemente demandas físicas, a capacidade para o trabalho relacionada às exigências mentais não estava prejudicada.

A predominância de lesões por acidentes e doenças músculo-esqueléticas provavelmente estava relacionada ao tipo de trabalho desenvolvido por esses trabalhadores, devido às exigências físicas no trabalho de higiene e limpeza.

A prevalência das doenças cardiovasculares e músculo-esqueléticas em grupo de trabalhadores finlandeses foi corroborada neste estudo. Os trabalhadores que realizavam demandas físicas ao longo desse estudo apresentaram freqüentemente alguma forma de doença, e ao responderem sobre a saúde, a perceberam como ruim ${ }^{(9)}$.

Num estudo com trabalhadores terceirizados da higiene e limpeza de um hospital público dentre as doenças referidas, com diagnóstico médico, estão: tendinite, dermatite, problemas psiquiátricos decorrentes do trabalho, bronquite, rinite e sinusite. Essas doenças podem estar relacionadas às condições de trabalho precárias tais como tarefas repetitivas, esforço físico contínuo e uso de produtos químicos para a limpeza ${ }^{(10)}$.

Os resultados obtidos com os trabalhadores do serviço de higiene e limpeza também foram semelhantes aos do estudo realizado com trabalhadores de enfermagem de um hospital universitário na cidade de São Paulo, no qual 67,5\% apresentaram lesões ou doenças músculo-esqueléticas, $18,8 \%$ problemas respiratórios, e 10,8\% tinham hipertensão $\operatorname{arterial}^{(11)}$.

Num estudo com trabalhadores de enfermagem de um Pronto-Socorro de um hospital universitário no interior de São Paulo verificou-se a associação entre as doenças e o comprometimento da capacidade para o trabalho, o ICT também foi utilizado nesse estudo. As doenças auto-referidas e referidas com diagnóstico médico foram: doença do sistema músculo-esquelético, doença do sistema cardiovascular, doença do sistema respiratório, doença do sistema neurológico e sensoriais. Esse achado sobre as doenças em trabalhadores da enfermagem corrobora os dados dessa pesquisa ${ }^{(12)}$.

As doenças auto-referidas mais freqüentes entre os trabalhadores do Serviço de Limpeza foram as músculoesqueléticas, 23,2\% da população, seguida pelas cardiovasculares, respiratórias e emocionais.

É importante ressaltar que as doenças respiratórias foram citadas por $15,9 \%$ dos trabalhadores. Uma possível interpretação desse dado seria a do ambiente de trabalho hospitalar, já que, há um risco de contaminação biológica, além do contato com produtos químicos utilizados no serviço de higiene e limpeza, que podem causar o comprometimento das funções respiratórias.
Os resultados obtidos sobre as doenças auto-referidas foram semelhantes aos de um estudo realizado com trabalhadores municipais na cidade de São Paulo no qual, as doenças mais citadas pelos trabalhadores na própria opinião foram as lesões nas costas, distúrbio emocional leve, lesão nos braços e mãos, doença superior das costas ou região do pescoço, obesidade e doença superior das cos$\operatorname{tas}^{(13)}$.

O aparecimento das doenças na própria opinião propiciou a seguinte reflexão: ou o trabalhador ainda não havia procurado um serviço de assistência à saúde para avaliação de sua queixa, ou os profissionais da área da saúde não as diagnosticaram.

As questões referentes aos recursos mentais, em geral, apresentaram-se com perspectivas positivas, no que diz respeito a sentir-se alerta e ativo, capaz de realizar atividades diárias e com esperança para o futuro. De acordo com esses resultados, foi possível verificar que há uma perspectiva do futuro positiva entre esses trabalhadores.

Houve associação entre a idade e a capacidade para o trabalho, quando foi considerado o grupo etário e o índice de capacidade para o trabalho, através do teste do quiquadrado ( $\mathrm{p}$ valor $<0,05$ ).

Ao realizar comparação com o grupo etário e as médias do Índice de Capacidade para o Trabalho, utilizando o Teste de Mann-Whitney ( $\mathrm{p}$ valor=0,0349), observou-se que havia uma diminuição da capacidade para o trabalho no grupo de maior idade, ou seja, de 50 a 60 anos. Esse grupo tinha 39 pontos de mediana no ICT, e o grupo de 30 a 49 anos, 34,5 pontos. Portanto, considerando-se a média e mediana, o grupo de mais idade apresentou um número maior de doenças, comprometendo assim, a capacidade para o trabalho.

Deve ser considerado ainda que os trabalhadores de mais idade estavam no mercado de trabalho há mais tempo que os mais jovens, além de não serem qualificados, e em trabalhos anteriores realizavam atividades que requeriam demanda física, é sugestivo pensar na perda da capacidade para o trabalho relacionada às condições de trabalho, e aos efeitos do processo de envelhecimento da população estudada.

O teste do qui-quadrado ( $\mathrm{p}$ valor $<0,05$ ) mostrou a associação entre os grupos etários e capacidade para o trabalho com relação às exigências físicas.

A comparação entre o grupo etário e as médias de doenças com diagnóstico médico mostrou que havia uma diferença significativa nos grupos, já que os trabalhadores entre 50 a 60 anos possuíam um número maior de doenças, em média 5,6 e o grupo de 30 a 49 anos, 3,1 (Teste de Mann-Whitney, p valor=0,0080). 
Estudo realizado na Finlândia mostrou que a capacidade para o trabalho esteve diminuída no decorrer do período de sua realização entre 1981-1992, estando fortemente associada com a idade e a carga de trabalho. Os trabalhadores de ambos os sexos acima de 51 anos com característica de trabalho com demanda física tiveram a capacidade para o trabalho diminuída ${ }^{(14)}$.

No entanto, o estudo com trabalhadores da enfermagem apontou que $13,2 \%$ da população apresentaram ICT moderado; $40,9 \%$, bom e $45,9 \%$, ótimo e a perda da capacidade para o trabalho esteve mais acentuada nos trabalhadores mais jovens. Assim, esse dado permite contradizer os dados sobre a capacidade para o trabalho dos trabalhadores da higiene e limpeza, já que o grupo de 50 a 60 anos teve o ICT comprometido, pois possuía um maior número de doenças ${ }^{(12)}$.

Um outro dado significante apresentado foi que os trabalhadores que possuíam doença músculo-esquelética diagnosticada tendiam a referir que precisavam diminuir o ritmo de trabalho (Teste de Mann-Whitney, p valor=0,0004).

Percebeu-se também, que houve relação entre o número de doenças e o prognóstico da capacidade para o trabalho no futuro próximo. Os trabalhadores que tinham um número maior de doenças tendiam a responder que era improvável, tendo como referência sua saúde, ter a capacidade de fazer o trabalho atual daqui a dois anos (Coeficiente de Correlação de Spearman $-0,55751$, p valor $=0,0001$ ).

De acordo com a metodologia do desenvolvimento do ICT, os trabalhadores que possuem um maior número de doenças tendem a ter a capacidade para o trabalho prejudicada. Esse achado foi corroborado por um estudo realizado em São Paulo com trabalhadores municipais, que mostrou que um maior número de doenças implicava no decréscimo do valor do ICT, além da capacidade física e mental e impedimento para o trabalho ${ }^{(15)}$.

Num estudo sobre educação continuada com trabalhadores terceirizados do serviço de higiene e limpeza de um hospital público verificou-se que durante as atividades educacionais realizadas por enfermeiras foram abordados temas como contaminação no ambiente hospitalar e uso de EPI (Equipamentos de Proteção Individual) $^{(16)}$.

No entanto, não foram salientados temas como a postura corporal no ambiente de trabalho e utilização adequada de equipamentos. Não houve discussão sobre as DORT (Doenças Osteomusculares Relacionadas ao Trabalho), e percebeu-se que a empresa terceirizada não oferecia equipamentos adequados como vassouras com cabo adaptável e baldes na altura ideal.

Também foram abordados os acidentes de trabalho, no entanto, as ocorrências foram referidas pela imprudência dos trabalhadores e não pela falta de informação e dificuldade de assimilar novas aprendizagens, já que a maioria dos trabalhadores possui baixa escolaridade.
Além das doenças apresentadas pelos trabalhadores da higiene e limpeza, há de considerar que os trabalhadores da área hospitalar estão expostos aos riscos biológicos e aos acidentes de trabalho com materiais perfurocortantes. Num estudo com trabalhadores de um hospital público verificouse que os maiores coeficientes de risco de acidentes foram de auxiliares de enfermagem, além de uma elevada subnotificação de acidentes de trabalho ${ }^{(17)}$.

Assim, pensar na saúde dos trabalhadores da higiene e limpeza requer olhar a centralidade do trabalho, ou seja, sua organização e suas condições. Para que ocorra a preservação da saúde e da capacidade para o trabalho é necessária a atuação interdisciplinar da equipe de saúde, para que se possa elaborar programas que visem a atenção à saúde desses trabalhadores.

\section{CONCLUSÕES}

Ao estudar o tema envelhecimento e capacidade para o trabalho é imprescindível que se considere a realidade brasileira, ou seja, as mudanças demográficas, as transformações na legislação previdenciária e no mundo do trabalho, sendo que essas, em geral, têm trazido repercussões nas condições de trabalho e saúde da população.

O estudo permitiu avaliar a capacidade para o trabalho entre os trabalhadores do Serviço de Higiene e Limpeza, sendo os resultados de grande importância para o acompanhamento da capacidade para o trabalho dessa população.

Ao analisar a capacidade para o trabalho desses trabalhadores, verificou-se, em alguns casos, que precisava ser melhorada ou restaurada, especialmente entre os trabalhadores mais velhos, e entre os que possuíam maior número de doenças. Isso porque, nesse estudo, a capacidade para o trabalho esteve relacionada à idade e ao número de doenças com diagnóstico médico.

O estudo sobre o tema envelhecimento e trabalho, na perspectiva da promoção da capacidade para o trabalho, é importante, na medida em que é possível realizar programas de prevenção de doenças relacionadas ao trabalho, e de mudanças na organização do trabalho, a fim de realizar a promoção da saúde e de qualidade de vida.

A realização desse trabalho pretende contribuir à equipe interdisciplinar da área da saúde, em especial à enfermagem, para que se possa dar visibilidade ao trabalho e à saúde dos funcionários da higiene e limpeza a fim de proporcionar programas que visem a manutenção e a promoção da saúde desses trabalhadores. É preciso olhar a área hospitalar como um local que também precisa "cuidar" da saúde dos trabalhadores, quaisquer que sejam eles. Dessa forma, o desenvolvimento de programas de saúde e de melhores condições de trabalho para os trabalhadores de serviços gerais, além de ser um desafio para a atuação do enfermeiro poderá ser um espaço de inovação da enfermagem. 


\section{REFERÊNCIAS}

1. Andrade CB, Monteiro-Cocco MI. Trabalho, envelhecimento e qualidade de vida: relatório final de iniciação científica. Campinas: SAE/Unicamp; 1999.

2. Silvestre JA, Kalache A, Ramos L, Veras RP. O envelhecimento populacional e o setor saúde. Arq Geriatr Gerontol. 1996;1(1):81-9.

3. Organización Mundial de la Salud (OMS). Él envejecimiento y la capacidad de trabajo: informe de un grupo de estudio de la OMS. Ginebra: OMS; 1993.

4. Tuomi K, Ilmarinen J, Martikainen R, Aalto L, Klockars M. Aging, work, life-style and work ability among Finnish municipal workers in 1981-1992. Scand J Work Environ Health. 1997;23 Suppl 1:58-65.

5. Costa G. Trabalho e envelhecimento. Rev Proteção. 2001 fev:46-55.

6. Nielsen J, Kopf T, Ambrosi B, Hopsu L, Søgaard K, Dittmann A, et al. Occupational health, individual capacities and work ability. In: Krüger D, Louhevaara V, Nielsen J, Scheider T. Risk assessment and preventive strategies in cleaning work. Hamburg: Polytechnical University Hamburg; 1997. p. 53-121.

7. Tuomi K, Ilmarinen J, Jahkola A, Katajarinne L, Tulkki A. Índice de Capacidade para o Trabalho. Tradução de Fischer FM. Helsinque: Instituto Finlandês de Saúde Ocupacional; 1997.

8. Almeida A Filho, Rouquayrol MZ. Introdução à epidemiologia moderna. $2^{\mathrm{a}}$ ed. Belo Horizonte: Coopmed; 1992.

9. Tuomi K, Ilmarinen J, Seitsamo J, Huuhtanen P, Martikainen R, Nygard C-H, et al. Summary of the Finnish research project (1981-1992) to promote the health and work ability of aging workers. Scand J Work Environ Health. 1997;23 Suppl 1:66-71.
10. Chillida MSP, Monteiro-Cocco MI. Saúde do trabalhador \& terceirização: perfil de trabalhadores de serviço de higiene e limpeza hospitalar. Rev Lat Am Enferm. 2004;12(2):271-6.

11. Fischer FM. Unveilling factors that contribute to fuctional aging among health care shiftworkers in São Paulo, Brazil. Experimental Aging Res. 2002;28(1):73-86.

12. Duran ECM, Monteiro-Cocco MI. Capacidade para o trabalho entre trabalhadores de enfermagem do pronto-socorro de um hospital universitário. Rev Lat Am Enferm. 2004;12(1):43-9.

13. Bellusci SM, Fischer FM. Envelhecimento funcional e condições de trabalho em servidores forenses. Rev Saúde Pública. 1999;33(6):602-9.

14. Ilmarinen J, Tuomi K, Klocars M. Changes in work abillity of active employees over an 11-year period. Scand J Work Environ Health. 1997;23 Suppl 1:49-57.

15. Monteiro MS. Envelhecimento e capacidade para o trabalho entre os trabalhadores brasileiros. [tese]. São Paulo: Faculdade de Saúde Pública, Universidade de São Paulo; 1999.

16. Monteiro MI, Chillida MSP, Bargas EB. Educação continuada em um serviço terceirizado de limpeza de um hospital universitário. Rev Lat Am Enferm. 2004;12(3):541-8.

17. Sarquis LM, Felli VE. Acidentes de trabalho com instrumentos perfurocortantes com trabalhadores de enfermagem. Rev Esc Enferm USP. 2002;36(3):222-30. 